NOTICE WARNING CONCERNING COPYRIGHT RESTRICTIONS:

The copyright law of the United States (title 17, U.S. Code) governs the making of photocopies or other reproductions of copyrighted material. Any copying of this document without permission of its author may be prohibited by law. 


\title{
Computational Models for Parallel Computers
}

\author{
H. T. Kung \\ August 28, 1987 \\ CMU-CS-88-164:
}

\section{Department of Computer Science \\ Carnegie Mellon University \\ Pittsburgh, Pennsylvania 15213}

\begin{abstract}
Computational models define the usage patterns of a computer. They can be used to derive the architecture of the machine, provide guidelines for programming tools, and suggest how the machine should be used in applications. Identifying computational models is especially important for parallel computers, since their architectures and usages are still not well understood in general.

This paper describes a number of computational models for parallel computers. These models characterize the communication pattems under which processors exchange their intermediate results during computation. Emphases are placed upon models for one-dimensional processor arrays, reflecting Carnegie Mellon's experiences with the Warp systolic array machine. These models include local computation, domain partition, pipeline, multifunction pipeline and ring.
\end{abstract}

This paper was prepared for the Royal Society Discussion Meeting on "Solving Scientific Problems on Multiprocessors", London, United Kingdom, December 9-10, 1987.

The research was supported in part by Defense Advanced Research Projects Agency (DOD) monitored by the Space and Naval Warfare Systems Command under Contract N00039-87-C-0251, and in part by the. Office of Naval Research under Contracts N00014-87-K-0385 and N00014-87-K-0533. 


\section{Introduction}

Many problems in science and technology are becoming so computationally demanding that conventional sequential computers can no longer provide the required computing power. Parallel computers have the potential to provide that power. A large number of parallel computers are commercially available. Shared memory parallel computers include MIMD machines such as Alliant, Encore, Sequent, and Cray X-MP. Distributed memory computers include MIMD machines such as Transputer, Warp, and Hypercube, and SIMD machines such as Connection Machine and DAP. Many more parallel machines of enhanced capabilities are under development. Successful use of parallel computers has been demonstrated in a number of application areas including scientific computing, signal and image processing, and logic simulation.

It is useful to develop models to capture important ways in which parallel computers are actually used in applications. These models can be used to derive architectures of new parallel machines, provide guidelines for programming tools, and suggest how each machine should be used in applications. There are roughly three stages in solving an application problem on a parallel computer.

Step 1: Application definition (e.g., by mathematical formula)

Step 2: Computation specification (e.g., by program)

Step 3: Computation on the parallel machine

Computational models described in this paper characterize the interprocessor communication behavior of Step 3 .

These computational models are based on our experiences in parallel algorithm design and parallel architecture development at Camegie Mellon. In 1984-87 Camegie Mellon developed a programmable systolic array machine called Warp, that has a one-dimensional (ID) array of 10 or more processing elements [3]. The machine is currently produced and marketed by General Electric Company. Anticipating the future need for integrated Warp systems, Carnegie Mellon and Intel Corporation have been developing a VLSI Warp chip, called the $i$ Warp chip. The $i$ Warp system will be available in 1989-90. Our work in Warp and iWarp has shown us the importance of being explicit about computational models in the development of a new parallel architecture as well as its applications and programming tools. The paper will mention some of these insights.

In this paper we describe computational models for 1D processor arrays. We use 1D processor arrays because their simple structure makes presentation easy and we have extensive applications experiences with the 1D array in Warp. It should be clear that the concepts presented here generalize to $2 \mathrm{D}$ or higher dimensional processor arrays, and other parallel computer architectures.

Section 2 provides background information on the Warp and $i$ Warp systems. Nine computational models for 1D processor arrays are presented in Section 3. Among them five models are frequently used on Warp. These are models corresponding to local computation, domain partition, pipeline, multifunction pipeline and ring. They will be discussed in more detail than the other models. The last section contains some concluding remarks. 


\section{Overview of Warp and $i$ Warp}

\subsection{Warp}

The Warp machine has three components - the Warp array, the interface unit, and the host, as depicted in Figure 1. We describe the machine only briefly here; details are available from a separate paper [3]. The Warp array performs the bulk of the computation. The interface unit handles the input/output between the array and the host. The host supplies data to and receives results from the array, in addition to executing the parts of the application programs that are not mapped onto the Warp array.

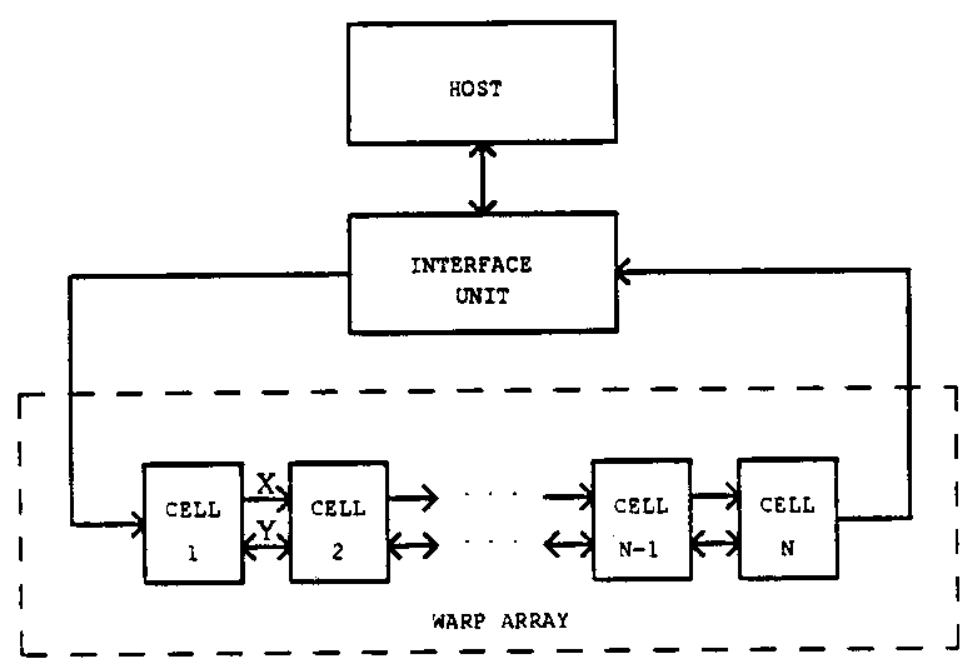

Figure 1. Warp machine overview

The Warp array is a 1D systolic array with identical processing elements called Warp cells. Data flow through the array on two communication channels ( $X$ and $Y$ ), as shown in Figure 1. The direction of the $Y$ channel is statically configurable at compile time. By configuring the $Y$ channel in the opposite direction from the $X$ channel, a ring interconnection can be formed inside the $\mathrm{ID}$ array. Another way to form a ring is to use the interface unit to connect the first and last cells of the array.

Each Warp ceil is implemented as a programmable horizontal micro-engine, with its own microsequencer and program memory. The cell data path includes a 5 MFLOPS floating-point multiplier (Mpy), a 5 MFLOPS floatingpoint adder (Add), a local memory, and two data input queues for the $X$ and $Y$ channels. All these components are connected through a crossbar. An output port of the crossbar can receive the value of any input port in each cycle. Via the crossbar the floating-point units can directly access data at the front of any input queue, and insert computed results at the end of any input queue of the next cell. Data at the front of any input queue can also be sent directly to the next cell. A (much) simplified description of the Warp cell data path is given in Figure 2.

A feature that distinguishes a Warp cell from many other processors of similar computation power is its high I/O 


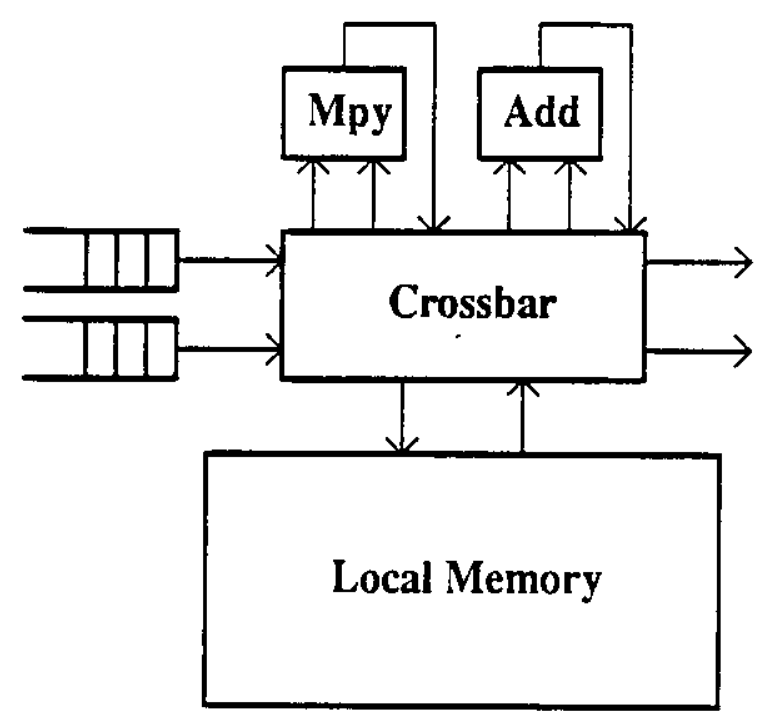

Figure 2. Warp cell data path (much simplied)

bandwidth - an important characteristic for systolic arrays. Each Warp cell can transfer up to 20 million words (80 Mbytes) to and from its neighboring cells per second. This high intercell communication bandwidth makes it possible to transfer large volumes of intermediate data between neighboring cells and support fine-grain parallelism on the Warp array.

The host consists of a Sun-3 workstation that serves as the master controller of the Warp machine, and a VME-based multi-processor "external host", so named because it is external to the workstation. The workstation provides a UNIX environment for running application programs. The external host controls the peripherals and contains a large amount of memory for storing data to be processed by the Warp array. Its dedicated processors transfer data to and from the Warp array and perform operations on the data, with low operating system overhead.

Warp programs are written in a high level Pascal-like language called W2, which is supported by an optimizing compiler $[6,18]$. To the application programmer, Warp is a ID array or a ring of simple sequential processors, communicating asynchronously. Based on the user's program for this abstract array or ring, the compiler generates code for the host, interface unit and Warp array automaticaily. W2 programs are developed in a Lisp-based programming environment supporting interactive program development and debugging. A C or Lisp program can call a W2 program from any UNIX computer on the local area network.

\section{2. $i$ Warp}

Carnegie Mellon and Intel are jointly developing a large VLSI chip, called the $i$ Warp chip, to implement an integrated version of the Warp cell. The $i$ Warp chip is a programmable processor capable of delivering at least 20 or 10 MFLOPS for single or double precision floating-point computations, respectively. This chip together with a local memory form the $i$ Warp cell, as depicted in Figure 3. The $i$ Warp cell is a powerful building-block cell for a variety of processor arrays, including $1 \mathrm{D}$ and $2 \mathrm{D}$ arrays. With recompilation, the $i$ Warp cell will be able to execute W2 programs originally written for the Warp cell. 


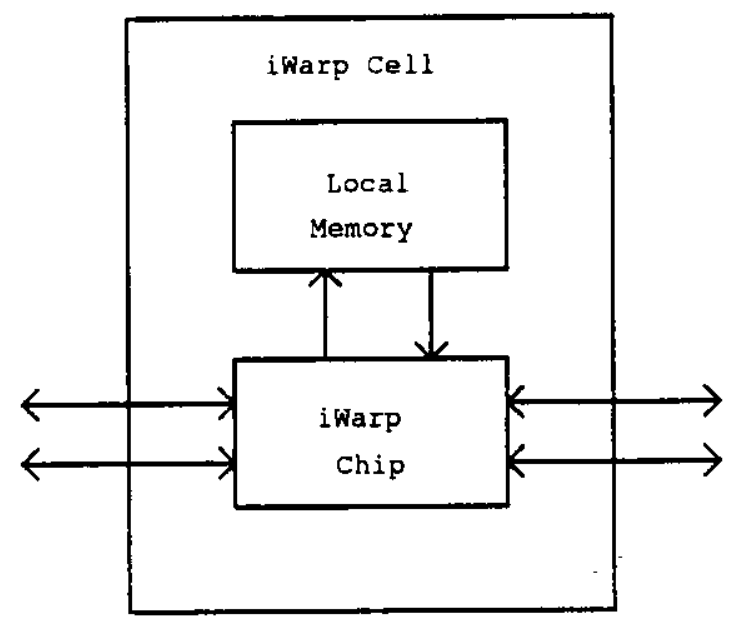

Figure 3. $i$ Warp cell consisting of $i$ Warp chip and local memory

The initial prototype $i$ Warp system will have an array of $72 i$ Warp cells, with a peak performance of at least 1,440 MFLOPS. To ensure that a large fraction of this peak performance can actually be realized in real applications, the $i$ Warp array supports the following features:

- large local memory for the cells (at least 24 address bits);

- high bandwidth intercell communication (320 Mbytes/sec);

- 2D or higher-dimensional interconnection; and

- on-chip message routing hardware.

Passing messages by a cell is handled by its routing hardware, and is transparent to its program. This implies that communication between non-neighboring cells can now be easily accomplished. 


\section{Computational Models}

We will describe the following computational models for $1 \mathrm{D}$ processor arrays:

1. local computation;

2. domain partition;

3. pipeline;

4. multifunction pipeline;

5. ring;

6. recursive computation;

7. divide-and-conquer;

8. query processing; and

9. task queue.

These models correspond to different ways in which cells interchange their intermediate results during computation. Under each model there may also be different ways in handling inputting and outputting for the processor array (see discussions below concerning the local computation model). Therefore the computational models are based on the communication behavior for intermediate results rather than input and output.

The current Warp system uses the first five models mostly, whereas the future $i$ Warp system will efficiently support all the models. Because of our experience with Warp, we will give more detailed descriptions for the first five models. The other models will only be briefly touched, mainly to indicate that there are other models which could be important for parallel computers to support.

In the diagrams, cells in a 1D processor array are denoted by square boxes, and named as cell 1 , cell $2, \cdots$, cell $N$ from left to right. Solid arrows denote data flows of intermediate results between cells.

\subsection{Local Computation Model}

The local computation model corresponds to the case where cells do not exchange their intermediate results during computation at all. Many computational problems have the property that elements in the output set are computed independently from each other. The use of the local computation model is natural in solving these problems on a parallel computer. In this model each output is computed entirely within a cell, and all the cells compute different outputs simultaneously. The main characteristic is that the entire computation for each output is done locally at a cell, i.e., the computation does not depend on intermediate results computed by other cells.

Various methods can be used to take care of the inputting and outputting for each cell. For example, before or during computation, the required input to a cell can be shifted in via the cells to the left, and during or after the computation the output produced by a cell can be shifted out via the cells to the right. This is depicted by Figure 4 , where dotted arrows denote the shift-in and shift-out paths for input and output, respectively. To achieve high performance, it is important that the I/O time and computation time can be overlapped as much as possible. 


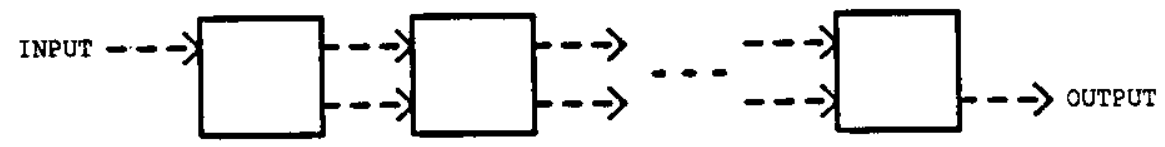

Figure 4. Local computation model, with input and output shifted in and out

Many image processing computations involve transforming an input image to an output image, using a kernel operator defined by, say, a $3 \times 3$ window. Figure 5 depicts such a transformation, with which each pixel in the output image depends on a neighborhood of the corresponding pixel in the input image. Clearly, all the pixels in the output image can be computed simultaneously and independently. Therefore the local computation model applies here. The figure illustrates that four cells can work on the four subregions of the output image independently, provided that the input pixels needed by each cell's computation are pre-stored in the cell. Note that cells computing adjacent subregions have overlapped input; the larger is the kemel the larger is the overlap.

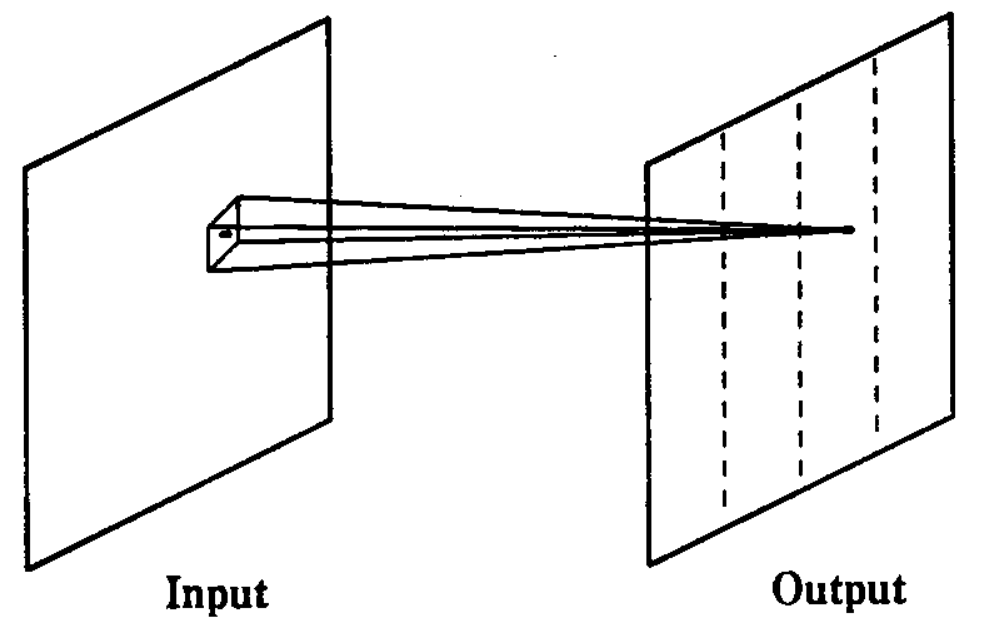

Figure 5. Local computation model for image processing using a kemel operator

As illustrated by the figure, the partitioning of the image processing task for the local computation model is straightforward. All that needs to be done is to partition the output image equally for all the cells. This partitioning has been automated-Camegie Mellon has developed a compiler called Apply, which can generate W2 programs for image processing computations based on kernel operators as described above, and other computations of similar kind [8].

Apply-generated W2 programs are able to overlap L/O with computation. While computing a row of pixels for the output image, a cell can output a previous row of pixels already computed and input a new row of pixels required for future computations. The Warp array supports this overlapping well, since the array has a high intercell communication bandwidth, and each cell is a horizontal micro-engine capable of performing several computation and I/O operations in each cycle. Because with Apply this overlapping is done automatically, Apply-generated Warp programs are often more efficient than the corresponding hand-generated code. 
There is another interesting form of overlapping input with computation for the local computation model. Although all the cells compute different parts of the output set, the cells may share some input. In this case the shared input may be pumped systolically from cell to cell during computation. In the following this is illustrated with a matrix multiplication example.

Given $n \times n$ matrices $A$ and $B$, we want to compute their product $C$ on a linear processor array of $k$ cells. We assume that $k$ is much less than $n$, and in the illustration below, $k=4$. We evenly partition columns of $B$ and $C$ as shown in Figure 6 (a). Using the local computation model, cell $i$ will compute the entries of submatrix $C_{i}$. As its inputs, cell $i$ needs $A$ and $B_{i}$. Therefore input $A$ is shared by all cells. Cell $i$ will first load entries of $B_{i}$ into its local memory. Then during computation, entries of matrix $A$ will be input to the left-most cell in the row-major ordering, and shifted to the right from cell to cell, as depicted in Figure 6 (b). Cell $i$ will perform inner products for all pairs of row and column in $A$ and $B_{i}$, respectively. (Each entry of $A$ will be input repeatedly as it will be used by each cell multiple times, one for each of the columns of $B$ that the cell has.) Each inner product involves reading in a row of $A$ from one of its input queues and a column of $B_{i}$ from the cell's local memory, and performing a sequence of multiply-accumulate operations. By shifting in entries of $A$ on-the-fly, each cell does not have to store the entire matrix. This can significantly save memory storage and access time for each cell [14].

(a)

[
A

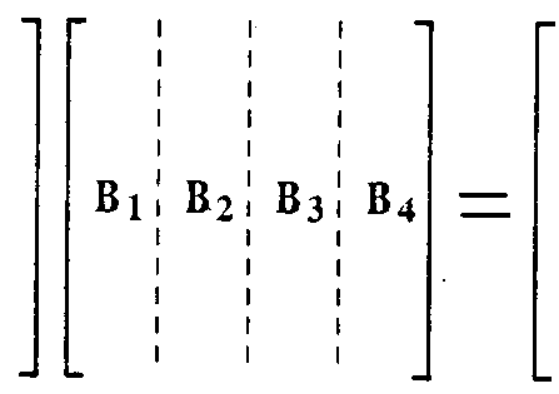

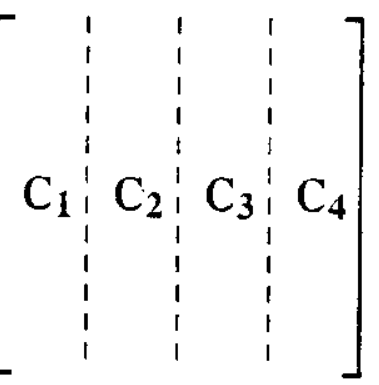

(b)

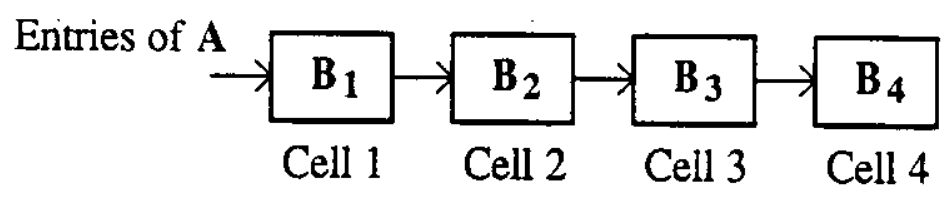

Figure 6. Matrix muitiplication: (a) partitioning of matrices $B$ and $C$, and (b) distribution of the resulting submatrices of $B$ to the cells; entries of $A$ moving to the right during computation

There are many other usage examples based on the local computation model. They include the discrete cosine transform [2] and the labeled histogram computation [17].

\subsection{Domain Partition Model}

For some applications the computation depicted in Figure 5 is repeated many umes; each time a new output image is computed based on the previous output image. This computational process, called successive relaxation [21, 22], is depicted in Figure 7, where the grids correspond to the images. 


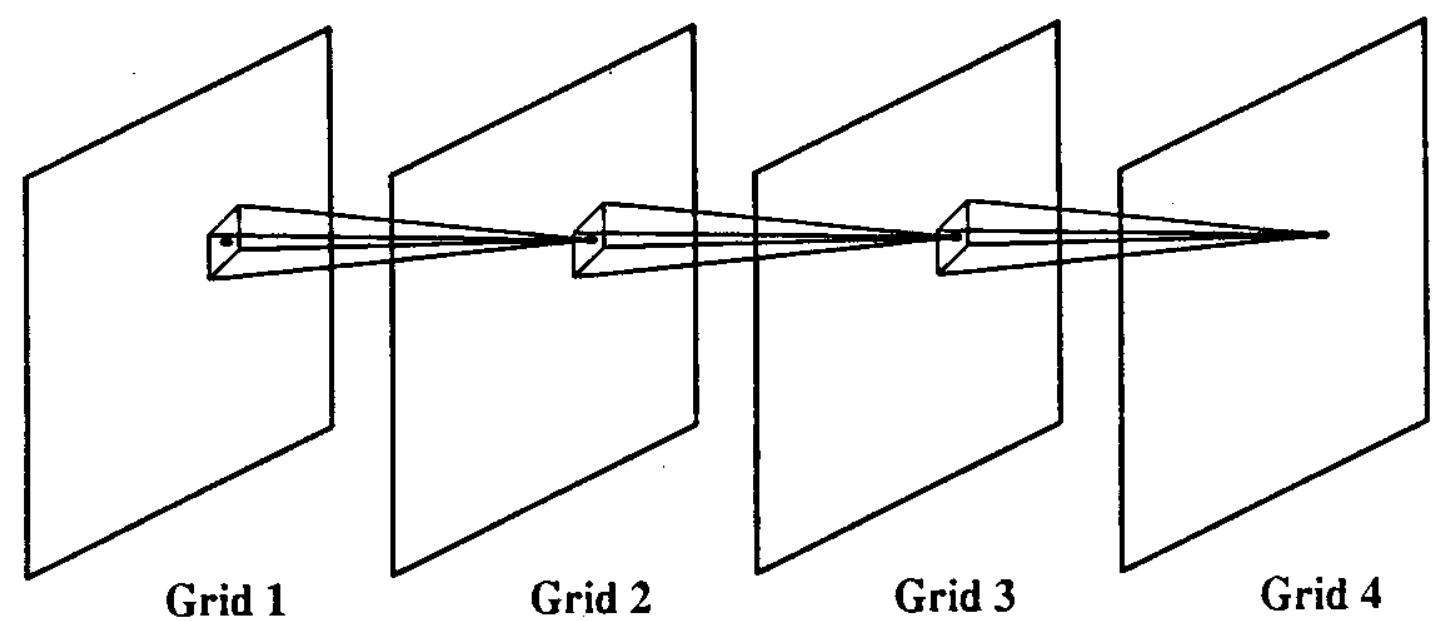

Figure 7. Successive relaxation

The successive relaxation process is often used in sciencific computing. Consider, for example, the solution of the following elliptic partial differential equations using successive overrelaxation [24]:

$$
\frac{\partial^{2} u}{\partial x^{2}}+\frac{\partial^{2} u}{\partial y^{2}}=f(x, y)
$$

The system is solved by repeatedly computing values of $u$ on a 2D grid using the following recurrence:

$$
u_{i, j}^{\prime}=(1-\omega) u_{i, j}+\omega \frac{f_{i, j}+u_{i, j-1}^{\prime}+u_{i, j+1}+u_{i+1, j}+u_{i-1, j}^{\prime}}{4}, \quad \text { where } \omega \text { is a constant parameter. }
$$

In the recurrence, values associated with location $(i, j)$ of the grid have indices $(i, j)$.

Suppose that the partitioning scheme of Figure 5 is used. Then when computing a new grid, each cell must import from its neighboring cells some of the values computed for the previous grid. The required bidirectional data flows between neighboring cells are shown in Figure 8.

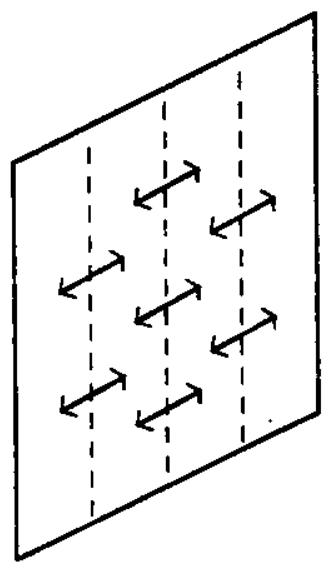

Figure 8. Bidirectional data flows for successive relaxation

With this example, the concept of the domain partition model can be easily introduced. The model arises when a 
problem domain (such as the grid space corresponding to an image, or to a finite difference or finite element modeling) is partitioned so that each cell handles a subdomain. This model differs from the local computation model in that each output is not computed entirely by a single cell. That is, once in a while the cell needs to receive intermediate results from its neighboring cells before it can proceed further with its computation. Figure 9 depicts the domain partition model.

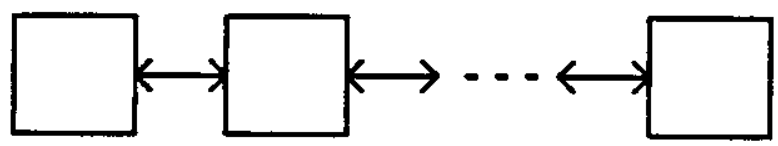

Figure 9. Domain partition model

There are many computations that can be naturally carried out using the domain partition model. Numerical simulations of properties of a physical object, formulated by either differential equations or Monte Carlo methods, can be partitioned along the physical space. A large file can be sorted on a 1D array by using the bi-directional communication to merge sublists sorted by individual cells. The merging can be done with only nearest neighbor communications, in a manner similar. to that used in the odd-even transposition sort [4]. Labeling of connected components in an image can be done by using the bidirectional communication to merge labels in the subimages computed by individual cells [17].

\subsection{Pipeline Model}

There is another (elegant) method to carry out the successive relaxation computation depicted in Figure 7 on a $1 \mathrm{D}$ array. This method uses pipelining. Instead of the data space, i.e., the grid, we partition along the time axis. That is, successive relaxation steps are done on successive cells. In the row-major ordering, each cell receives inputs from the preceding cell, performs its relaxation step, and outputs the results to the next cell. Consider for example the successive overrelaxation computation described in Section 3.2. While a cell is performing the $k^{\text {th }}$ relaxation step on row $i$, the preceding and next cells perform the $(k-1)^{\text {th }}$ and $(k+1)^{\text {th }}$ relaxation steps on rows $i+2$ and $i-2$, respectively. Thus, in one pass of the $u$ values through a $k$-cell processor array, $k$ relaxation steps are performed. This process is repeated until convergence is achieved. In a similar way we can implement many other iterative methods such as Jacobi and Gauss-Seidel methods in a pipelined manner.

In this pipeline model, the computation for each output is partitioned into a sequence of identical stages, and cell $i$ is responsible for stage $i$. A characteristic of this model is that cell $i+1$ uses computed results of cell $i$, as depicted in Figure 10. Intermediate results move in one direction and final results emerge from the last cell. I/O and computation are automatically overlapped; this is a major advantage of the model. The pipeline model is natural when implementing systolic algorithms where the partial results move from cell to cell and get updated at each cell they pass $[12,15]$.

Under the pipeline model, cell $i+1$ cannot start its operation until cell $i$ completes at least a stage of computation. 


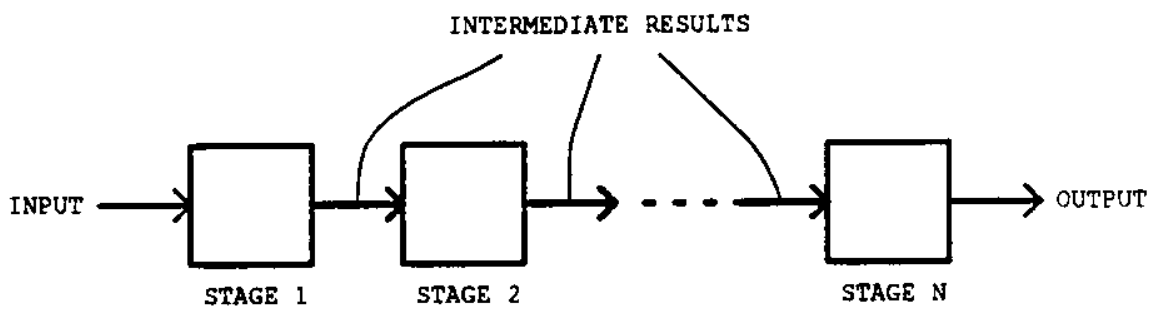

Figure 10. Pipeline model

Thus for this model minimizing the latency between the starting times of adjacent cells is a major concern. This is in contrast with the domain partition model, for which the starting time of a cell does not depend upon any computed results of other cells.

For some computations the pipeline model represents the only efficient parallel implementation. To see such a case, consider a variant of the image processing task depicted in Figure 5. For this variant, in computing the value of each point, the new values of its neighbors will be used whenever possible. Suppose that using a $3 \times 3$ window, the computation follows the row-major ordering. Then computing the value of each new point uses the new values of the left neighbor and the upper three neighbors, which were computed earlier. Local computation and domain partition models will not work here since subregions of the image cannot be computed independently from each other. A way of using the pipeline model is that cell $i$ computes values of points in row $i$ in the left to right order. Cell $i$ is pre-stored with values of points in rows $i$ and $i+1$. During computation, a copy of each new value cell $i$ computes is sent to cell $i+1$. Note that cell $i+1$ can start its computation as soon as cell $i$ has computed the values of the first two points in row $i$. We have implemented a version of this pipeline computation on Warp to solve a path planning problem using a dynamic programming technique [5].

\subsection{Multifunction Pipeline Model}

A single computation may involve a series of subcomputations each performing a different function. If these functions can be chained together on a 1D array, then a one-pass execution of the entire computation will be possible. This is the basic idea of the multifunction pipeline model [7]. In this model, the 1D array is a pipeline of several groups, each consisting of a number of cells devoted to a different function. The number of ceils in each group is adjusted so that every group will take about the same time, in order to maximize the pipeline throughput.

This model is illustrated in the following example, which is a laser radar simulation implemented on Warp:

Step 1: For every 1024-point input block, perform a 1024-point complex FFT. Partition each FFT output into 30 overlapped 256 -element subsequences.

Step 2: For each of the 30 256-element subsequences, perform the following operations:

(i) multiply each element by a weight, which is a complex number;

(ii) perform a 256-point complex inverse FFT;

(iii) compute the amplitude of each element in the output subsequences.

Step 3: Threshold the resulting $30 \times 256$ image using $3 \times 3$ windows. 


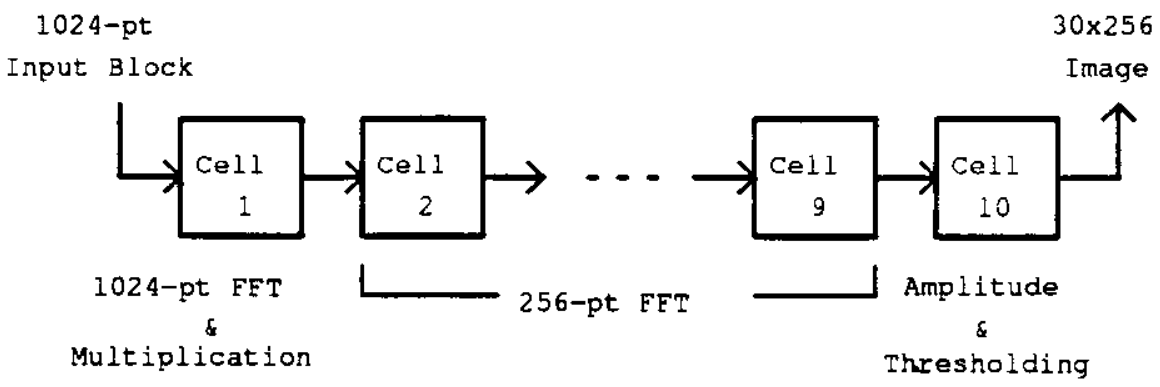

Figure 11. Multifunction pipeline model to implement a radar simulation on Wap

These steps are implemented with consecutive segments of the Warp array, as depicted in Figure 11.

Figure 12 illustrates another possible use of the multifunction pipeline model in implementing the geometry system portion of 3D computer graphics. The first cell performs the matrix multiplications, the next three cells do clipping, and the last cell does the scaling operation. Three cells are devoted to clipping as it requires more arithmetic operations than either matrix multiplication or scaling [10].

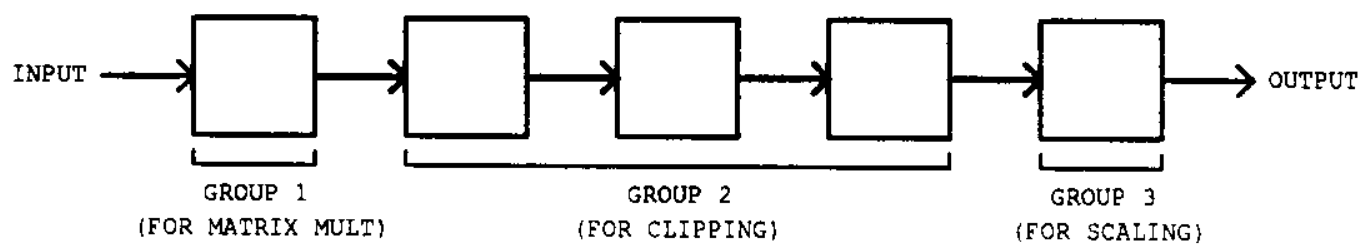

Figure 12. Multifunction pipeline model to implement a geometry system

The multifunction pipeline model is useful when a computation requires a number of small functions, each of which is not large enough to make an effective use of all the cells in a $1 \mathrm{D}$ array. Concatenating these functions in a chain offers an opportunity to use more cells effectively. Also, for some computations, it is inherent that one or few cells must perform functions different from the rest For example, when performing a $2 D$ convolution on a $1 D$ array, some cells need to buffer a row of image and none of the other cells need to do that [13]. For some computations, the first and last cells of a ID array carry out special functions such as interface with the outside world or preparation of data for the next phase of computation on the array. An example of this is a neural network simulation on Warp, where only the last cell performs weight updates based on weight changes computed by other cells [19].

To support the multifunction model, the processor array must allow heterogeneous programming, that is, different programs to be executed at different cells at a given time. Further, the rate of the input to a group may not be compatible to that of the output from the preceding group. Thus some buffering and flow control mechanisms need to be provided between each pair of cells. For the Warp array, all cells can be individually controlled. and dedicated hardware queues capable of performing flow control are available between adjacent cells.

In summary, the multifunction model differs from the pipeline model described earlier in that cells are now 
allowed to perform different functions. This flexibility in the usage offers the opportunity of effectively using a large number of cells in a 1D array.

\subsection{Ring}

A 1D array becomes a ring when the first cell is connected to the last cell. In the ring model intermediate results flow on a ring of cells.

An important usage of the ring model is the implementation of a large "logic" array of logical cells, under the pipeline model, with a small "physical" array of physical cells. One implementation is to have each physical cell handle a group of consecutive logical cells as depicted in Figure 13 (a). This will incur a large latency between the starting times of two adjacent physical cells, as the latency will be the sum of all the latencies incurred by those logical cells which are assigned to a physical cell. Another implementation is to use the physical array in multiple passes to simulate the function of the logical array, as depicted in Figure 13 (b). This multiple pass scheme can be implemented with a ring as shown in Figure 13 (c). The ring is formed by using a queue to connect the last physical cell to the first. The queue can store outputs from the last physical cell while the first is still busy in doing its computation for the current pass. This ring scheme incurs the minimum latency between the starting times of two adjacent physical cells.

(a)

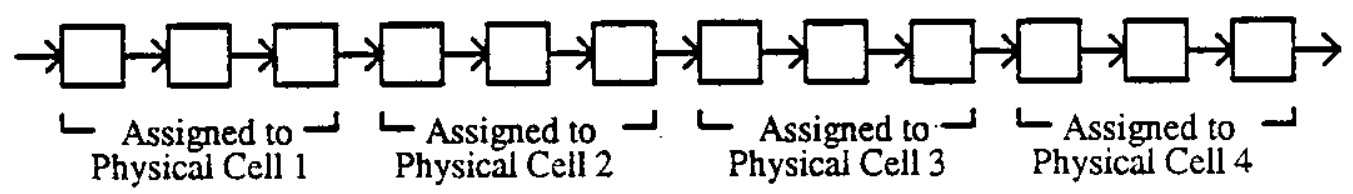

(b)

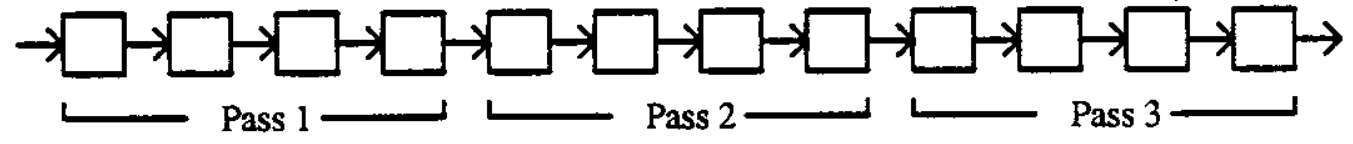

(c)

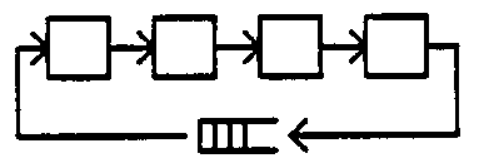

Figure 13. Implementing a large pipeline with a small physical array:

(a) each physical cell is assigned to a set of consecutive logical cells, (b) using the physical array in multiple passes, and (c) using a ring to implement the multiple passes on the physical array

Another major use of the ring model is in the implementation of broadcasting. Many computational problems involve multiple levels of computation as depicted in Figure 14 (a). Each value in a level depends on all the values computed in the previous level. For example, in the figure to compute $b_{1}$ in level 2 we need all the values in level 1 , as indicated by the lines connecting $b_{1}$ with $a_{1}, a_{2}, a_{3}$ and $a_{4}$. Therefore all the values computed in a level need to be broadcast to all the cells which will be computing values in the next level. An example of such a computational problem is the back propagation neural network simulation [23], for which levels of computation correspond to 
(a)

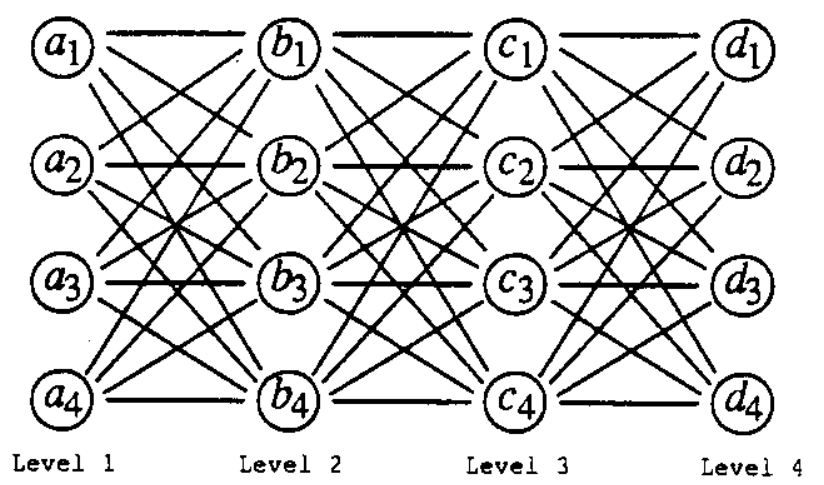

(b)

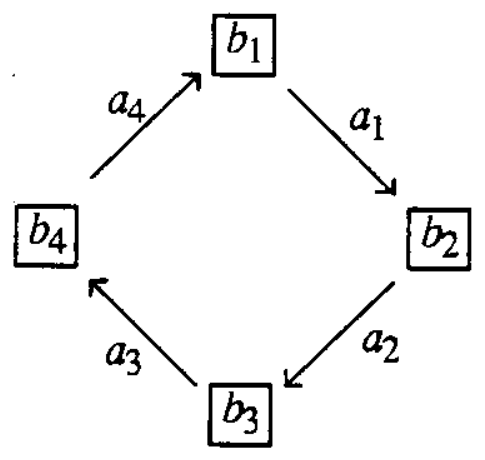

Figure 14. (a) Multilevel computation where results in one level are broadcast to the next level, and (b) using the ring model to implement the broadcasting

layers of the neural network.

The ring structure can implement the broadcasting in a natural way, provided that the computation for each value is commutative and associative so that inputs in the previous level can be combined in any order. Figure 14 (b) illustrates the idea, by considering how values in level 1 can be sent to cells computing values in level 2 . Assume that every value in a layer is computed by a separate cell, and for each $i$ the cell which computes $a_{i}$ will also compute $b_{i}$. Then by pumping the $a_{i}$ 's around the ring for a full cycle, as shown in Figure 14 (b), cell $i$ (for every $i$ ) will be able to meet all the $a_{i}$ 's so it will have all the inputs to compute $b_{i}$. The computation of $b_{i}$ will occur on-the-fly as each $a_{i}$ passes by. Therefore computation and I/O are totally overlapped.

\subsection{Recursive Computation Model}

Recursive computations are those where results of the computation are used for computing future results. Examples are recursive filtering [11], solution of triangular linear systems [16], and QR-decomposition [9]. By flowing outputs that were previously computed against the flow of intermediate results that are currently being computed, recursive computations can be implemented. The important feature of the recursive computation model is the propagation of outputs in the opposite direction of intermediate results, as illustrated by Figure 15. 


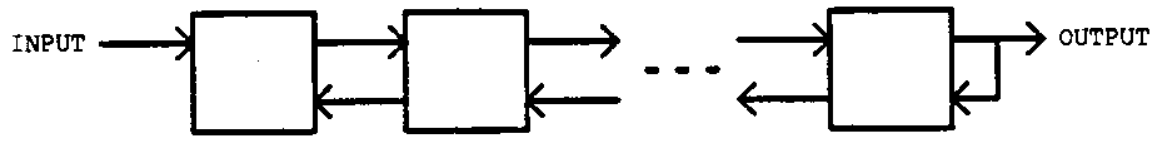

Figure 15. Recursive computation model

\subsection{Divide-and-conquer Model}

Divide-and-conquer is a fundamental technique in algorithm design [1]. Under this design paradigm, we solve a problem by (1) partitioning it into subproblems of nearly equal size, (2) solving all the subproblems, and (3) merging the solutions to the subproblems; this procedure is applied recursively to all the subproblems. Because of this recursion, this partitioning scheme distinguishes itself from others used, in, for example, the local computation and domain partition models. Figure 16 illustrates the divide-and-conquer model. Each subproblem is carried out by one cell or a set of consecutive cells. When a (sub)problem is partitioned into subproblems or solutions to subproblems are merged, communications between cells that are either 1-apart, 2-apart, $\cdots$, or N/2-apart take place. These communications are depicted by solid arrows in the figure.

(a)
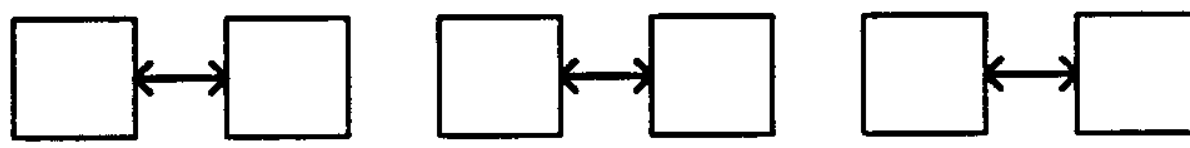

(b)
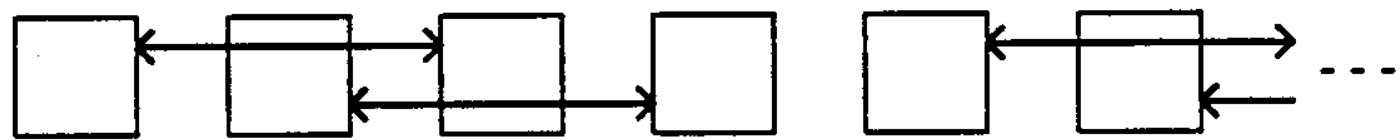

Figure 16. Divide-and-conquer model: (a) 1-apart communication and (b) 2-apart communication

The divide-and-conquer model for example can be used in sorting, and various geometric problems such as computing convex hulls [20].

\subsection{Query Processing Model}

A $1 D$ array can be used to process queries. One way to do this is to have the database partitioned evenly among the cells. Then queries are passed to all the cells. Every cell looks at the arriving query and outputs its reply to the query. The query processing model is depicted in Figure 17.

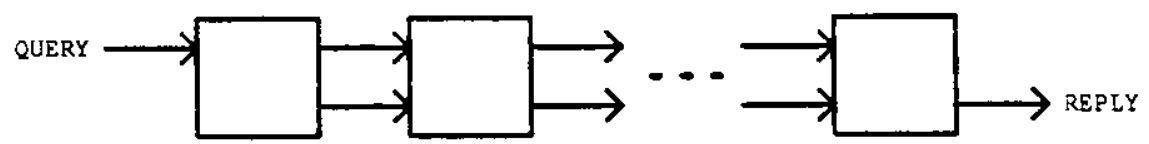

Figure 17. Query processing model

Consider for example the problem of looking for a table in an image. The particular table we are searching for is defined as having a rectangular top, which will appear as a parallelogram in the image. Initially, we do not know anything about the position of the table, except an upper bound on the size of its bounding square in the image. After extracting features such as lines and edges from the image, we partition it into regions whose sizes are at least 
that of the bounding square for the table. We assign each region to a cell. To balance the computational load between the cells, we define the regions so that there are about the same number of features associated with each region. Regions assigned to the cells are properly overlapped to ensure that the entire table is contained in at least one region. All the cells can work in parallel on their own regions to respond to the query:

"list all sets of four lines that form a parallelogram".

Given the response to this query, the host or the cell that controls the searching process can predict the position of other sides of the table, and produce queries such as:

"list parallel lines with a given orientation",

to find the other sides of the table.

The query processing model requires that the cells operate asynchronously, as when responding to a query they may have to perform different amounts of computations and may produce variable amounts of outputs.

\subsection{Task Queue Model}

For all of the preceding models, cells work together for a common task, whether they are tightly coupled (as in the pipeline model) or loosely coupled (as in the local computation or domain partition model). In contrast, the task queue model allows different cells to work on different tasks in one application. More precisely, a free cell can be dynamically assigned to execute any task in a task queue maintained by a cell or the host, as depicted by Figure 18. Cells operate in a totally independent and asynchronous manner. Using this model, dynamic load balancing between cells is possible. The major concern in the implementation of this model is to minimize the latency between when a cell becomes free and when it starts doing a new task sent from the task queue. To use the cell effectively, this latency should not be larger than the time for the cell to execute the task.

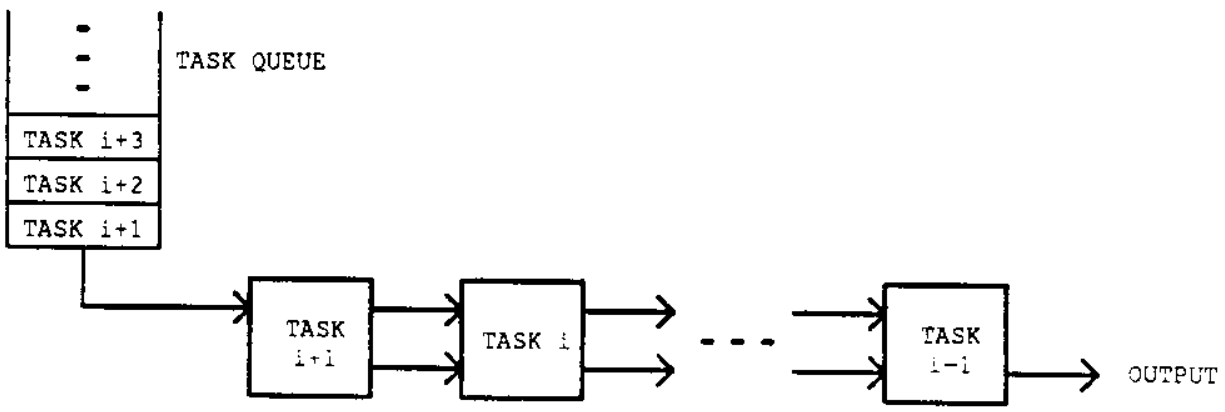

Figure 18. Task queue model

The task queue model will be efficiently supported by the $i$ Warp system. The on-chip message router at each cell will allow flexible communication between the cell (or host) that maintains the task queue and ocher cells. The communication will have low latency because of the available high bandwidth intercell communication channels. 


\section{Concluding Remarks}

In this paper we have informally described a number of computational models for 1D processor arrays. Among these models, local computation, domain partition, pipeline, multifunction pipeline, and ring are frequently used by the Warp users. We have found that in terms of these models various applications usages of the machine can be easily described. Also, we can discuss how architectural features support these models. For examples, the 1D systolic array is natural for the pipeline model; and the routing hardware is needed for the efficient support of the divide-and-conquer or task queue model. Moreover, these models provide a way to classify programming tools for the automatic generation of parallel programs. For example, the Apply programming tool is to generate parallel code for the local computation model. There are several ongoing research projects at Carnegie Mellon intended to generate parallel programs for the other computational models such as the pipeline model.

For these reasons, we believe that computational models need to be made as explicit as possible in parallel computing. This paper represents an initial attempt to identify some of the models that seem to be important. Further work is needed to expand this set of models, and characterize them more precisely. Eventually, notations need to be developed to represent computational models. 


\section{Acknowledgment}

Many of the ideas presented in this paper were inspired by work done under the Warp project at Carnegie Mellon. The author is especially indebted to those members of the project, including F. Bitz, G. Gusciora, H. Ribas, P. S. Tseng, and J. Webb, for their implementation of some of the applications examples discussed in this paper. 


\section{References}

1. Aho, A., Hopcroft, J.E. and Ullman, J.D.. The Design and Analysis of Computer Algorithms. Addison-Wesley, Reading, Massachusetts, 1975.

2. Annaratone, M., Arnould, E., Kung, H. T. and Menzilcioglu, O. Using Warp as a Supercomputer in Signal Processing. Proceedings of ICASSP 86, IEEE, 1986, pp. 2895-2898.

3. Annaratone, M., Arnould, E., Gross, T., Kung, H. T., Lam, M., Menzilcioglu, O. and Webb, J. A. "The Warp Computer: Architecture, Implementation and Performance". IEEE Transactions on Computers C-36, 12 (December 1987), 1523-1538.

4. Baudet, G. and Stevenson, D. "Optimal Sorting Algorithms for Parallel Computers". IEEE Transactions on Computers C-27, 1 (January 1978), 84-87.

5. Bitz, F. and kung, H. T. Path planning on the Warp computer: using a linear systolic array in dynamic programming. Proceedings of SPIE Symposium, Vol. 826, Advanced Algorithms and Architectures for Signal Processing II, Society of Photo-Optical Instrumentation Engineers, August, 1987. The final version is to appear in International Journal of Computer Mathematics (1988).

6. Gross, T. and Lam, M. Compilation for a High-performance Systolic Array. Proceedings of the SIGPLAN 86 Symposium on Compiler Construction, ACM SIGPLAN, June, 1986, pp. 27-38.

7. Gross, T., Kung. H.T., Lam, M. and Webb, J. Warp as a Machine for Low-level Vision. Proceedings of 1985 IEEE International Conference on Robotics and Automation, March, 1985, pp. 790-800.

8. Hamey, L. G. C., Webb, J. A., and Wu, I. C. Low-level Vision on Warp and the Apply Programming Model. In Parallel Computation and Computers for Artificial Intelligence, Kluwer Academic Publishers, 1987, pp. 185-199. Edited by J. Kowalik.

9. Heller, D.E. and Ipsen, I.C.F. Systolic Networks for Orthogonal Equivalence Transformations and Their Applications. Proceedings of Conference on Advanced Research in VLSI, Massachusetts Institute of Technology, Cambridge, Massachusetts, January, 1982, pp. 113-122.

10. Hsu, F.H., Kung, H.T., Nishizawa, T. and Sussman, A. Architecture of the Link and Interconnection Chip. Proceedings of 1985 Chapel Hill Conference on VLSI, Computer Science Department, The University of North Carolina, May, 1985, pp. 186-195.

11. Kung, H.T. Let's Design Algorithms for VLSI Systems. Proceedings of Conference on Very Large Scale Integration: Architecture, Design, Fabrication, Califomia Institute of Technology, January, 1979, pp. 65-90.

12. Kung, H.T. "Why Systolic Architectures?". Computer Magazine 15, I (Jan. 1982), 37-46.

13. Kung, H.T. Systolic Algorithms for the CMU Warp Processor. Proceedings of the Seventh International Conference on Pattern Recognition, International Association for Pattern Recognition, 1984, pp. 570-577. A revised revion appears as Chapter 3 in Systolic Signal Processing Systems, edited by E. E. Swartzlander, Jr., pp. 73-95, New York, Marcel Dekker, 1987.

14. Kung, H. T. Systolic Communication. Proceedings of the Intemational Conference on Systolic Arrays, May, 1988, pp. 695-703.

15. Kung, H.T. and Leiserson, C.E. Systolic Arrays (for VLSD). Sparse Matrix Proceedings 1978, Society for Industrial and Applied Mathematics, 1979, pp. 256-282. A slightly different version appears in Introduction to VLSI Systems by C. A. Mead and L. A. Conway, Addison-Wesley, 1980, Section 8.3, pp. 37-46..

16. Kung, H.T. and Leiserson, C.E. Systolic Arrays (for VLSI). Sparse Matrix Proceedings 1978, Society for Industrial and Applied Mathematics, 1979, pp. 256-282.

17. Kung, H. T. and Webb, J. A. "Mapping Image Processing Operations onto a Linear Systolic Machine". Distributed Computing 1, 4 (1986), 246-257.

18. Lam, M. S. A Systolic Array Optimizing Compiler. Ph.D. Th., Carnegie Mellon University, May 1987. 
19. Pomerleau, D. A., Gusciora, G. L., Touretzky, D. S. and Kung, H. T. Neural Network Simulation at Warp Speed: How We Got 17 Million Connections Per Second. 1988 IEEE Intemational Conference on Neural Networks, July, 1988.

20. Preparata, F.P. and Shamos, M.I.. Computational Geometry: In Introduction. Springer-Verlag, New York, 1985.

21. Rosenfeld, A. Iterative methods in image analysis. Proceedings of the IEEE Computer Society Conference on Pattern Recognition and Image Processing, Intemational Association for Pattem Recognition, 1977, pp. 14-18.

22. Rosenfeld, A., Hummel, R. A., and Zucker, S. W. "Scene labelling by relaxation operations". IEEE Trans. on Systems, Man, and Cybernetics SMC-6 (June 1976), 420-433.

23. Rumelhart, D. E., Hinton, G. E., and Williams, R. J. Learning Internal Representations by Error Propagation. In Rumelhart, D. E. and McClelland, J. L., Ed., Parallel Distributed Processing: Explorations in the Microstructure of Cognition. Vol. I: Foundations, Bradford Books/MIT Press, Cambridge, MA., 1986, pp. 318-362.

24. Young, D.. Iterative Solution of Large Linear Systems. Academic Press, New York, 1971. 